\title{
Utilização do cateter central de inserção periférica em neonatos: análise da indicação à remoção
}

\author{
Use of the central catheter for peripheral insertion in newborns: analysis of indication for \\ removal
}

Maria Aparecida Baggio ${ }^{1}$, Maycon Hoffmann Cheffer ${ }^{1}$, Mayara Aparecida Passaura da Luz ${ }^{1}$, Michelle de Marchi Sanches $^{1}$, Rosilene Berres ${ }^{1}$

Objetivo: analisar a utilização do cateter central de inserção periférica em neonatos. Métodos: estudo retrospectivo, documental. Para coleta de dados, utilizou-se de instrumento para registro e acompanhamento de cateter central de inserção periférica. Análise realizada por meio de estatísticas descritivas para obtenção de frequências absolutas, relativas, valores mínimos e máximos, desvios-padrão e médias e teste de quiquadrado para k-proporções para variáveis qualitativas. Resultados: foram analisados 383 registros. 0 cateter foi indicado, principalmente, para administração de antibioticoterapia (46,5\%). 0 vaso mais acessado foi a veia cefálica $(23,5 \%)$. A complicação mais incidente foi de progressão $(6,8 \%)$, com prevalência de remoção por término de tratamento/indicação (25,3\%). Conclusão: cateter utilizado, principalmente, para administração de antibioticoterapia; veia cefálica mais acessada, com uso de Fentanil para atenuar dor e desconforto; progressão, complicação mais incidente; e maior índice de remoção por término de tratamento/indicação.

Descritores: Cateterismo Venoso Central; Cateterismo Periférico; Unidades de Terapia Intensiva; Cuidados de Enfermagem; Enfermagem Neonatal.

Objective: to analyze the use of the central catheter for peripheral insertion in newborns. Methods: retrospective, documentary study. For data collection, we used an instrument to record and follow the central catheter for peripheral insertion. Analysis performed using descriptive statistics to obtain absolute, relative, minimum and maximum values, standard and mean deviations, and chi-square test for K-proportions for qualitative variables. Results: we analyzed 383 records. The catheter was mainly indicated for administration of antibiotic therapy (46.5\%). The most accessed vessel was the cephalic vein (23.5\%). The most frequent complication was progression (6.8\%), with prevalence of removal due to end of treatment/indication $(25.3 \%)$. Conclusion: catheter used mainly for antibiotic therapy administration; cephalic vein mostly accessed, using fentanyl to mitigate pain and discomfort; progression, mostly incident complication; and higher removal rate due to end of treatment/indication.

Descriptors: Catheterization, Central Venous; Catheterization, Peripheral; Intensive Care Units; Nursing Care; Neonatal Nursing.

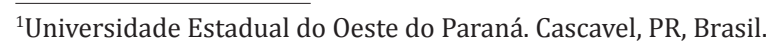




\section{Introdução}

0 acesso intravenoso em neonatos hospitalizados em unidades de cuidados intensivos se baseia na indicação clínica. Atualmente, tem-se variedade de opções disponíveis, favorecida pelos avanços tecnológicos $^{(1)}$ Dentre estas, tem-se o cateter central de inserção periférica, com primeiros relatos de uso na década de 1970, nos Estados Unidos ${ }^{(2)}$.

0 cateter central de inserção periférica é um dispositivo de acesso vascular, inserido em uma extremidade, como veia basílica ou cefálica, e avançado até que a ponta esteja posicionada no terço médio da veia cava superior ou inferior ${ }^{(2-4)}$ Uma das indicações é a terapia intravenosa de longa duração, superior a seis dias, utilizada em Neonatologia e Pediatria ${ }^{(1,5)}$.

A inserção e manipulação por enfermeiros é amparada pelo Conselho Federal de Enfermagem ${ }^{(3-6)}$, sendo a manutenção de acesso venoso, particularmente em neonatos clinicamente instáveis, desafio aos profissionais responsáveis pela assistência ${ }^{(1)}$.

A inserção, a manutenção e o controle da infecção do cateter central de inserção periférica requerem práticas assistenciais que promovam a segurança do paciente, com menor exposição à dor, bem como redução de riscos e complicações inerentes ao procedimento ${ }^{(4)}$. Neste sentido, entender os processos que envolvem a inserção do cateter central de inserção periférica e a avaliação de práticas de manutenção pela enfermagem, em unidades de terapia intensiva neonatal, podem fornecer estratégias para boas práticas e melhores resultados no cuidado de recém-nascidos em uso do cateter ${ }^{(7)}$, a priori, evitando intercorrências e remoção precoce do cateter.

Ao considerar o exposto e que a inserção do cateter central de inserção periférica é realizada em unidade de cuidados intensivos neonatais, de hospital universitário do Oeste do Paraná, Brasil, desde 2008, com utilização de instrumento para registro e acompanhamento de cateter central de inserção periférica, sem qualquer avaliação acerca da prática de enfer- magem, questionou-se: o que os instrumentos para registro e acompanhamento de cateter central de inserção periférica apontam acerca da indicação, inserção e remoção do cateter? Logo, objetivou-se analisar a utilização do cateter central de inserção periférica em neonatos.

\section{Métodos}

Estudo retrospectivo, de levantamento documental, realizado em Hospital Universitário do Estado do Paraná, Brasil, com população de 1.052 neonatos internados em unidade de terapia intensiva neonatal, de julho de 2009 a julho de 2014. Destes, foram selecionados para amostra os neonatos que fizeram uso de cateter central de inserção periférica (inseridos e removidos na instituição do estudo) e continham no prontuário o instrumento para registro e acompanhamento de cateter central de inserção periférica, totalizando amostra de 383 instrumentos de análise de neonatos.

A coleta de dados foi realizada de julho de 2015 a janeiro de 2016, por meio de instrumento para registro e acompanhamento de cateter central de inserção periférica, elaborado por enfermeiros habilitados do campo do estudo para inserção do cateter, presente no prontuário impresso dos neonatos.

Foram variáveis analíticas: identificação do neonato (sexo, idade, peso, diagnóstico); dados de indicação, inserção (data, produto para degermação, duração do procedimento, número de tentativas de punção, vaso puncionado, medidas para controle da dor, intercorrências, posicionamento da ponta do cateter) e remoção do cateter (data, motivo). A ausência de preenchimento de variáveis do instrumento condicionou a consulta de registros contidos nos prontuários impressos e eletrônicos para localizar informações faltantes, não havendo perdas dos instrumentos da amostra.

A análise dos dados foi realizada por meio de estatísticas descritivas das variáveis qualitativas (fre- 
quência absoluta, frequência relativa e limites inferior e superior do intervalo de confiança (IC) a 95\%) e das variáveis quantitativas (média, desvio padrão e limites inferior e superior do IC a 95\%). Além disso, foram realizados testes de qui-quadrado para k-proporções para variáveis qualitativas. Os testes estatísticos foram realizados com auxílio do software estatístico XLSTAT $^{\circledR}$, com exceção dos intervalos de confiança, realizados através do software estatístico $\mathrm{R}$.

O estudo foi aprovado pelo Comitê de Ética em Pesquisa da Universidade Estadual do Oeste do Paraná, conforme parecer no 861.914/2014 e Certificado de Apresentação para Apreciação Ética no 35908114.9.0000.0107.

\section{Resultados}

Dos 383 instrumentos para registro e acompanhamento de cateter central de inserção periférica analisados, 57 (14,9\%) estavam totalmente preenchidos e $326(85,1 \%)$ com uma ou mais variáveis sem preenchimento.

Os neonatos da amostra, internados na unidade de terapia intensiva neonatal, apresentaram, em média, $14 \pm 28$ dias de idade (IC: 11 - 17), com peso médio de $1.836 \pm 1.035$ gramas (IC: 1.816 - 2.043) e com tempo de internamento médio de $44 \pm 52$ dias (IC: 39 - 49).

Observou-se que a média de uso do cateter, desde a inserção até a remoção, foi de $16 \pm 13$ dias (IC: 14 - 17), e a duração do procedimento foi de $38 \pm 33$ minutos (IC: 35 - 42). Em relação às tentativas de punção, a média foi de $3 \pm 2$ tentativas (IC: 3 - 4). Na distribuição dos sexos, 220 (57,0\%) neonatos eram do sexo masculino e 163 (43,0\%) do feminino.

No tocante ao diagnóstico médico, a maior parte dos problemas estava relacionada à prematuridade, 147 (38,4\%); e sistema respiratório, 90 (23,5\%). No concernente à indicação para uso do cateter, identificaram-se 178 (46,5\%) indicações para antibioticoterapia e $90(23,5 \%)$ para nutrição parenteral total $(\mathrm{p}<0,001)$ (Tabela 1).
Tabela 1 - Frequências absolutas e relativas de diagnóstico médico e indicação para uso do cateter, em neonatos internados em unidade de terapia intensiva neonatal

\begin{tabular}{lccc}
\hline Variáveis & $\mathbf{n}(\mathbf{\%})$ & IC (95\%)* & p-valor \\
\hline Diagnóstico médico & & & \\
Prematuridade & $147(38,4)$ & $35,4-41,4$ & \\
Sistema respiratório & $90(23,5)$ & $20,9-26,1$ & \\
Sistema digestório & $51(13,3)$ & $11,2-15,4$ & \\
Sepse & $36(9,4)$ & $7,6-11,2$ & \\
Sistema nervoso & $21(5,5)$ & $4,1-6,9$ & $<0,001$ \\
Baixo peso & $13(3,4)$ & $2,3-4,5$ & \\
Más formações & $10(2,6)$ & $1,6-3,6$ & \\
Sistema circulatório & $6(1,6)$ & $0,8-2,3$ & \\
Sistema músculo esquelético & $3(0,8)$ & $0,2-1,3$ & \\
Sistema urinário & $2(0,5)$ & $0,1-1$ & \\
Informação ausente & $4(1,0)$ & $0,4-1,7$ & \\
Indicação do cateter & & & \\
Antibioticoterapia & $178(46,5)$ & $43,4-49,6$ & \\
Nutrição parental total & $90(23,5)$ & $20,9-26,1$ & $<0,001$ \\
Prematuridade & $16(4,2)$ & $2,9-5,4$ & \\
Cirurgias em geral & $4(1,0)$ & $0,4-1,7$ & \\
Outras indicações & $67(17,5)$ & $15,1-19,8$ & \\
Informação ausente & $28(7,3)$ & $5,7-8,9$ & \\
\hline Fonte: Prontuários de hospital universitário. *IC: Intervalo de confiança; †Tes- \\
te Qui-quadrado para proporção & & &
\end{tabular}

Os vasos sanguíneos mais puncionados foram as veias cefálica $90(23,5 \%)$ e safena $54(14,1 \%)$. Outros vasos (auricular esquerda, axilar, acessória, cefálica acessória, femural, jugular anterior, jugular posterior, poplítea, radial, retro auricular, temporal, veia femural) aparecem como prevalentes, 97 (25,3\%) $(p<0,001)$ (Tabela 2). As intercorrências durante a inserção do cateter central de inserção periférica foram pouco frequentes, sendo a dificuldade de progressão a mais incidente, $26(6,8 \%)(p<0,001)$. Em relação aos motivos da remoção do cateter, a maior frequência foi de término de tratamento/indicação, 97 (25,3\%) $(\mathrm{p}<0,001)$. No entanto, o não registro do motivo de remoção foi identificado em 128 (33,4\%) dos prontuários e instrumentos analisados (Tabela 2). 
Tabela 2 - Frequências absolutas e relativas de vasos puncionados, intercorrências e motivos da remoção do cateter de neonatos internados em unidade de terapia intensiva neonatal

\begin{tabular}{|c|c|c|c|}
\hline Variáveis & n (\%) & IC (95\%)* & p-valor \\
\hline \multicolumn{4}{|l|}{ Vaso puncionado } \\
\hline Veia cefálica & $90(23,5)$ & $20,9-26,1$ & \multirow{8}{*}{$<0,001$} \\
\hline Veia safena & $54(14,1)$ & $11,9-16,3$ & \\
\hline Veia basílica & $41(10,7)$ & $8,8-12,6$ & \\
\hline Veia jugular & $21(5,5)$ & $4,1-6,9$ & \\
\hline Veia axilar & $6(1,6)$ & $0,8-2,3$ & \\
\hline Veia braquial & $5(1,3)$ & $0,6-2$ & \\
\hline Outros & $97(25,3)$ & $22,6-28$ & \\
\hline Informação ausente & $69(18,0)$ & $15,6-20,4$ & \\
\hline \multicolumn{4}{|l|}{ Intercorrências } \\
\hline Não tem & $338(88,3)$ & $86,3-90,2$ & \multirow{5}{*}{$<0,001$} \\
\hline Dificuldade na progressão & $26(6,8)$ & $5,2-8,3$ & \\
\hline Sangramento & $10(2,6)$ & $1,6-3,6$ & \\
\hline Dificuldade de acesso & $7(1,8)$ & $1-2,7$ & \\
\hline Outras & $2(0,5)$ & $0,1-1$ & \\
\hline \multicolumn{4}{|l|}{ Motivo da remoção } \\
\hline Término do tratamento & $97(25,3)$ & $22,6-28$ & \multirow{10}{*}{$<0,001$} \\
\hline Alta hospitalar & $51(13,3)$ & $11,2-15,4$ & \\
\hline Obstrução & $37(9,7)$ & $7,8-11,5$ & \\
\hline Óbito & $31(8,1)$ & $6,4-9,8$ & \\
\hline Sinais flogísticos & $11(2,9)$ & $1,8-3,9$ & \\
\hline Retirada acidental & $8(2,1)$ & $1,2-3$ & \\
\hline Ruptura & $7(1,8)$ & $1-2,7$ & \\
\hline Infiltração & $3(0,8)$ & $0,2-1,3$ & \\
\hline Outros & $10(2,6)$ & $1,6-3,6$ & \\
\hline Informação ausente & $128(33,4)$ & $30,5-36,3$ & \\
\hline
\end{tabular}

Do total dos cateteres analisados, 235 (61,3\%) dos pacientes tiveram os cateteres removidos na própria unidade de terapia intensiva neonatal $(\mathrm{p}<0,001)$. Quanto à degermação da pele para inserção do cateter, em $109(28,4 \%)$ casos, identificou-se a clorexidina alcoólica 2,0\%; e em 105 (27,4\%), a clorexidina alcoólica a $4,0 \%(p<0,001)$.

Referente à solicitação de cultura da ponta do cateter, evidenciou-se maior frequência de não solicitação $314(82,0 \%) \quad(p<0,001)$. Das solicitações de cultura da ponta do cateter, dois $(15,4 \%)$ casos apresentaram resultado positivo, sendo que um exame constatou bactérias e leveduras; e outro, apenas leveduras. Foram oito $(61,5 \%)$ com resultados negativos à solicitação de cultura da ponta do cateter e três $(23,1 \%)$ que não tiveram o resultado da análise preenchido (Tabela 3).

Tabela 3 - Frequências absolutas e relativas de unidade de remoção do cateter, degermação da pele, cultura da ponta do cateter, medidas para atenuar dor e desconforto, radiografia e posicionamento da ponta do cateter

\begin{tabular}{|c|c|c|c|}
\hline Variáveis & n (\%) & IC $(95 \%)^{*}$ & p-valor ${ }^{\dagger}$ \\
\hline \multicolumn{4}{|l|}{ Unidade de remoção do cateter } \\
\hline Unidade de terapia intensiva neonatal & $235(61,3)$ & $58,3-64,4$ & \multirow{5}{*}{$<0,001$} \\
\hline Unidade de cuidados intermediários & $34(8,9)$ & $7,1-10,6$ & \\
\hline Outro hospital & $11(2,9)$ & $1,8-3,9$ & \\
\hline Unidade de terapia intensiva pediátrica & $4(1,0)$ & $0,4-1,7$ & \\
\hline Informação ausente & $99(25,9)$ & $23,1-86$ & \\
\hline \multicolumn{4}{|l|}{ Degermação da pele } \\
\hline Clorexidina alcoólica 2\% & $109(28,4)$ & $25,7-31,3$ & \multirow{5}{*}{$<0,001$} \\
\hline Clorexidina alcoólica 4\% & $105(27,4)$ & $24,7-30,2$ & \\
\hline $\begin{array}{l}\text { Clorexidina sem identificação de con- } \\
\text { centração }\end{array}$ & $96(25,1)$ & $22,4-27,8$ & \\
\hline Solução alcoólica 70\% & $57(14,9)$ & $12,7-17,1$ & \\
\hline Informação ausente & $16(4,2)$ & $2,9-5,4$ & \\
\hline \multicolumn{4}{|l|}{ Solicitação de cultura da ponta do cateter } \\
\hline Não solicitação & $314(82,0)$ & $79,6-84,4$ & \multirow{3}{*}{$<0,001$} \\
\hline Solicitação & $13(3,4)$ & $2,3-4,5$ & \\
\hline Informação ausente & $56(14,6)$ & $12,4-16,8$ & \\
\hline \multicolumn{4}{|l|}{ Medicações } \\
\hline Fentanil endovenoso & $151(39,4)$ & $36,4-42,5$ & \multirow{7}{*}{$<0,001$} \\
\hline Paracetamol via oral & $37(9,7)$ & $7,8-11,5$ & \\
\hline Dormonid endovenoso & $33(8,6)$ & $6,9-10,4$ & \\
\hline Morfina endovenosa & $1(0,3)$ & $-0,1-0,6$ & \\
\hline Outras & $15(3,9)$ & $2,7-5,1$ & \\
\hline Não usou & $92(24,0)$ & $21,4-26,7$ & \\
\hline Informação ausente & $54(14,1)$ & $11,9-16,3$ & \\
\hline \multicolumn{4}{|l|}{ Medidas não farmacológicas } \\
\hline Não utilizada & $347(90,6)$ & $88,8-92,4$ & \multirow{5}{*}{$<0,001$} \\
\hline Glicose $50,0 \%$ via oral & $22(5,7)$ & $4,3-7,2$ & \\
\hline Amamentação & $1(0,3)$ & $-0,1-0,6$ & \\
\hline Outras & $7(1,8)$ & $1-2,7$ & \\
\hline Informação ausente & $6(1,6)$ & $0,8-2,3$ & \\
\hline \multicolumn{4}{|l|}{ Radiografia } \\
\hline Sim & $321(83,8)$ & $81,5-86,1$ & \multirow[t]{2}{*}{$<0,001$} \\
\hline Número ausente & $62(16,2)$ & $13,9-18,5$ & \\
\hline \multicolumn{4}{|l|}{ Posicionamento da ponta do cateter } \\
\hline Posicionamento central & $284(74,1)$ & $71,4-76,9$ & \multirow{3}{*}{$<0,001$} \\
\hline Posicionamento periférico & $24(6,3)$ & $4,8-7,8$ & \\
\hline Informação ausente & $75(19,6)$ & $17,1-22$ & \\
\hline
\end{tabular}


Na avaliação dos medicamentos via endovenosa para atenuar a dor e o desconforto, a maioria recebeu Fentanil 151 (39,4\%), não sendo utilizadas medidas não farmacológicas para atenuar a dor e o desconforto em $347(90,6 \%)$ casos $(\mathrm{p}<0,001)$ (Tabela 3$)$.

A radiografia torácica para confirmar a posição central do cateter foi realizada em $321(83,8 \%)$ dos instrumentos e prontuários analisados $(\mathrm{p}<0,001)$. Quanto ao posicionamento da ponta do cateter, após realização da radiografia, a posição central foi registrada em $284(74,1 \%)$ casos ( $\mathrm{p}<0,001)$ (Tabela 3).

\section{Discussão}

Considerou-se limitação do estudo o não preenchimento de inúmeras variáveis dos instrumentos e a ausência de registros nos prontuários analisados.

Os resultados apontaram a importância do uso de instrumentos/protocolos para suprir lacunas entre evidências científicas e a prática de enfermagem, visto que indicação, inserção, manutenção e remoção do cateter central de inserção periférica devem estar baseados em protocolos institucionais, desenvolvidos a partir da sistematização da assistência de enfermagem $^{(5)}$.

Os dados sinalizaram maior incidência do uso do cateter central de inserção periférica em neonatos do sexo masculino e peso inferior a 2.000 gramas. As complicações da prematuridade (síndrome do desconforto respiratório ou doença da membrana hialina, asfixia perinatal, sepse, entre outros) ${ }^{(8)}$, cujos fatores de risco são a própria prematuridade e o baixo peso ao nascimento, podem justificar as proporções identificadas $^{(9)}$.

0 peso inferior a 2.500 gramas, no momento de inserção do cateter, é previsor de infecção, pelo uso do dispositivo, seguido pelo tempo de uso em dias e realização de reparo do cateter ${ }^{(3,10-11)}$. No entanto, a retirada do cateter, por sinais flogísticos, mostrou-se pouco incidente na unidade investigada. Todavia, é prevalente o não preenchimento do motivo da remoção.
A inserção do cateter central de inserção periférica ocorre, principalmente, em neonatos com idades de menos de três até mais de 61 dias vida, sendo utilizado comumente em neonatos internados em ambiente intensivo ${ }^{(3,12)}$.

Quanto aos diagnósticos de neonatos em uso do cateter central de inserção periférica, confirma-se serem mais incidentes, como neste estudo, diagnósticos de prematuridade, afecção dos sistemas respiratório e cardíaco e má formação fetal ${ }^{(3)}$. Diagnósticos relacionados ao baixo peso, à afecção do sistema digestório, ao choque ou sepse, identificados em outros $\operatorname{achados}^{(10-11)}$, aparecem pouco ou não incidentes neste estudo.

O cateter central de inserção periférica é inserido, geralmente, para substituir o cateter venoso umbilical ou pela fragilidade da rede venosa. A indicação deste é definida pela equipe de saúdee, usualmente, é adequado para terapias de longa duração ${ }^{(3)}$, podendo ser de várias semanas até seis meses para administração de nutrição parenteral, infusão de medicações vesicantes, irritantes, vasoativas, soluções hiperosmolares, quimioterápicos, antimicrobianos, repetidas transfusões sanguíneas. Neste estudo, a prevalência dos diagnósticos de prematuridade e problemas relacionados ao sistema respiratório justifica as indicações de cateter, principalmente para antibioticoterapia e nutrição parenteral total, corroborando com evidência científica ${ }^{(4)}$.

Diferentes estudos apontam as veias basílica, cefálica e safena como preferencialmente puncionadas para inserção do cateter central de inserção periférica ${ }^{(10-11)}$, além de outros vasos, como a veia mediana cubital e jugular(2). Entretanto, diretrizes internacionais recomendam para punção as veias basílica, cefálica, mediana cubital e braquial, sendo as duas primeiras indicadas por apresentarem menor dificuldade de posicionamento do cateter, menor número de válvulas e maior calibre, possuírem anatomia favorável e permitirem fácil troca de curativos ${ }^{(13)}$. Recomenda-se evitar áreas de articulação e priorizar veias distais e superficiais, exceto em condições de emergência, ci- 
rurgias e lesões locais ${ }^{(14)}$.

As características anatômicas, fisiológicas e cognitivas inerentes à faixa etária do neonato podem influenciar no sucesso da punção, bem como prematuridade, existência de doença crônica, tratamento cirúrgico prolongado, terapia intravenosa periférica prolongada e uso de medicamentos vesicantes ${ }^{(15)}$, sendo, portanto, necessário o enfermeiro ter conhecimento acerca da anatomia, fisiologia da pele e do sistema venoso e capacidade de avaliar condições clínicas e ou cirúrgicas específicas de cada neonato.

As tentativas de punção e o tempo de duração do procedimento podem estar relacionados às particularidades da inserção do dispositivo em neonatos e, também, às intercorrências durante o procedimento. As chances de infecção aumentam, conforme se elevam as tentativas de punção, além de expor o neonato à maior condição de dor ${ }^{(16)}$.

Durante a inserção do cateter, podem ocorrer intercorrências, como dificuldade de progressão do cateter, sangramento, hematomas, risco para formação de trombose e flebite ${ }^{(15)}$. Neste estudo, as principais intercorrências foram dificuldade de acesso, progressão do cateter e sangramento.

Quanto aos motivos de remoção do cateter central de inserção periférica, confirma-se a prevalência de remoção eletiva, por término da terapia intravenosa e ou alta da unidade de terapia intensiva ${ }^{(12)}$, além de extração por complicações, como infiltração, extravasamento, tromboflebite, infecção local ${ }^{(15)}$, entre outros. Estes últimos, pouco evidentes na unidade de terapia intensiva neonatal estudada. Contudo, o não preenchimento dessa informação no prontuário foi prevalente aos demais motivos.

0 tempo de permanência do cateter correspondeu à média dos resultados de pesquisas nacionais e internacionais, as quais demonstraram que a maioria dos cateteres permaneceu por período superior a cinco dias, com variação de um a 38 dias $^{(12,16)}$.

Para degermação da pele, identifica-se utilização de clorexidina alcóolica 2,0\% e álcool 70,0\%, respectivamente, como primeira e segunda escolha, conforme recomendações internacionais e uso em outras realidades ${ }^{(16-17)}$. Porém, salienta-se considerável ausência de registro quanto ao tipo de clorexedina (alcóolica e ou degermante) e respectivas concentrações ${ }^{(18)}$ para degermação da pele de neonatos.

A baixa incidência de solicitação de cultura da ponta do cateter, possivelmente, está relacionada à remoção do cateter, somente quando houver sinais de infecção relacionada ao cateter ${ }^{(3)}$. Logo, a baixa incidência de retirada do cateter por sinais flogísticos relaciona-se à baixa incidência de infecção. Ademais, as duas hemoculturas positivas dos cateteres retirados por suspeita de infecção em corrente sanguínea relacionadas ao cateter do estudo são compatíveis com resultados de baixa incidência de culturas positivas em cateter central de inserção periférica ${ }^{(16)}$.

0 uso adequado de intervenções ambientais, comportamentais e farmacológicas pode reduzir a dor em recém-nascidos, durante a instalação do cateter central de inserção periférica. Intervenções não farmacológicas de primeira escolha são a sacarose e sucção não nutritiva (chupeta) ou leite humano. Além disso, a administração de solução de glicose (glucose), o tratamento individualizado com limitação de estímulos ambientais, posicionamento lateral, reforço facilitado (segurar braços e pernas em posição flexível), musicoterapia, contato pele a pele materno ou paterno, a redução de luz e ruído e presença parental, também, podem ser utilizadas ${ }^{(19)}$.

Confirmam-se o uso de glicose, além do leite materno, as medidas de contato e a utilização de glicose associado ou não ao leite materno ${ }^{(16)}$ como medidas não farmacológicas eficazes para alívio da dor durante o procedimento. Contudo, é prevalente a não utilização de ações para prevenção e controle da dor durante a inserção do cateter por meio de estratégias não farmacológicas, mesmo que evidências apontem a eficácia destas ${ }^{(20)}$. As estratégias farmacológicas para analgesia e sedação são as mais utilizadas, via endovenosa, geralmente em bolus, com uso de Fentanil(11), Midazolam $^{(14)}$, Dipirona e Paracetamol ${ }^{(20)}$.

Identifica-se elevado número de instrumentos 
e prontuários sem confirmar a posição da ponta do cateter, a qual pode ser confirmada por radiografia torácica ou ultrassonografia. Destaca-se que o uso de ultrassonografia por enfermeiros, na prática clínica, para guiar a punção venosa periférica em neonatos, garante maior segurança na execução do procedimento ${ }^{(2,19)}$.

\section{Conclusão}

O cateter foi principalmente utilizado para administração de antibioticoterapia, sendo a veia cefálica a mais acessada, com uso de Fentanil para atenuar dor e desconforto; a complicação mais incidente foi a de progressão; e o maior índice de remoção, por término de tratamento/indicação. Contudo, identificou-se fragilidade no preenchimento do instrumento para registro e acompanhamento do cateter central de inserção periférica, da indicação à remoção do cateter, o que pode comprometer o planejamento e a avaliação da assistência de enfermagem.

\section{Colaborações}

Baggio MA, Cheffer MH, Luz MAP e Sanches MM colaboraram com a concepção, projeto, análise e interpretação dos dados, redação do artigo e revisão crítica relevante do conteúdo intelectual e aprovação da versão final a ser publicada. Berres R contribuiu com redação do artigo, revisão crítica relevante do conteúdo intelectual e aprovação final da versão a ser publicada.

\section{Referências}

1. Vera SO, Sousa GN, Araújo SNM. The work of nurses in the practice of inserting and maintaining the PICC: a literature integrative review. Reon Facema [Internet]. 2015 [cited May 7, 2019]; 1(1):47-53. Available from: http://www.facema.edu.br/ojs/ index.php/ReOnFacema/article/download/9/12

2. Onofre PSC, Pedreira MLG, Barros DP, Peterlini MAS. Cateter intravenoso central de inserção periférica guiado por ultrassonografia: relato de experiência. Rev Soc Bras Enferm Ped [Internet]. 2016 [citado 2019 Jan 10]; 16(2):96-9. Disponível em: http://www.sobep.org.br/revista/images/ stories/pdf-revista/vol16-n2/vol_16_n_2-relato_ de_experiencia_4.pdf

3. Costa P, Paiva ED, Kimura AF, Castro TE. Risk factors for bloodstream infection associated with peripherally inserted central catheters in neonates. Acta Paul Enferm. 2016; 29(2):161-8. doi: http:// dx.doi.org/10.1590/1982-0194201600023

4. Santo MKD, Takemoto D, Nascimento RG, Nascimento AM, Siqueira É, Duarte CT, et al. Peripherally inserted central venous catheters: alternative or first choice vascular access? J Vasc Bras. 2017; 16(2):104-12. doi: http://dx.doi. org/10.1590/1677-5449.011516

5. Oliveira CR, Neves ET, Rodrigues EC, Zamberlan KC, Silveira A. Peripherally inserted central catheter in pediatrics and neonatology: possibilities of systematization in a teaching hospital. Esc Anna Nery. 2014; 18(3):379-85. doi: http://dx.doi. org/10.5935/1414-8145.20140054

6. Conselho Federal de Enfermagem. Resolução $n^{\circ} 258 / 2001$ [Internet]. 2001 [citado 2019 ago. 15]. Disponível em: http://cofen.gov.br/resoluocofen-2582001_4296.html

7. Pedreira MLG. Obstruction of peripherally inserted central catheters in newborns: prevention is the best intervention [editorial]. Rev Paul Pediatr. 2015; 33(3):255-7. doi: dx.doi.org/10.1016/j. rpped.2015.05.003

8. Sousa DS, Sousa Júnior AS, Santos ADR, Melo EV, Lima SO, Almeida-Santos MA, et al. Morbidity in extreme low birth weight newborns hospitalized in a high risk public maternity. Rev Bras Saúde Matern Infant. 2017; 17(1):139-47. doi: http:// dx.doi.org/10.1590/1806-93042017000100008

9. Gonzaga ICA, Santos SLD, Silva ARV, Campelo V. Prenatal care and risk factors associated with premature birth and low birth weight in the a capital in the Brazilian Northeast. Ciênc Saúde Coletiva. 2016; 21(6):1965-74. doi: http://dx.doi. org/10.1590/1413-81232015216.06162015

10. Rangel UV, Gomes Junior SCS, Costa AMAM, Moreira MEL. Variables associated with central peripheral insertion catheter infection in high risk newborns. Rev Latino-Am Enfermagem. 2014; 
22(5):842-7. doi: dx.doi.org/10.1590/01041169.3481 .2488

11. Lui AML, Zilly A, França AFO, Ferreira E, Toninato APC, Silva RMM. Care and limitations in the management of the peripherally inserted central catheter in neonatology. Rev Enferm Centr O Min. 2018; 8:e1918. doi: http://dx.doi.org/10.19175/ recom.v7i0.1918

12. Jantsch LB, Neves ET, Arrué AM, Kegler JJ, Oliveira CR. Utilização do cateter central de inserção periférica em neonatologia. Rev Baiana Enferm [Internet]. 2014 [citado 2019 Fev 22]; 28(3):24451. Disponível em: http://www.portalseer.ufba. br/index.php/enfermagem/article/view/10109/

13. Araújo FL, Manzo BF, Costa ACL, Corrêa AR, Marcatto JO, Simão DAS. Adherence to central venous catheter insertion bundle in neonatal and pediatric units. Rev Esc Enferm USP. 2017; 51:e03269. doi: dx.doi.org/10.1590/s1980-220x2017009603269

14. Phillips LD, Gorski LA. Manual of IV Therapeutics: evidence-based practice for infusion therapy [Internet]. 2014 [cited jun 13, 2019]. Available from: http://iranianfamilyphysicians.com/images/ book/Lynn_D_Phillips_MSN_RN_CRNI,_.pdf

15. Danski MTR, Mingorance P, Johann DA, Vayego SA, Lind J. Incidence of local complications and risk factors associated with peripheral intravenous catheter in neonates. Rev Esc Enferm USP. 2016; 50(1):22-8. doi: http://dx.doi.org/10.1590/ S0080-623420160000100003
16. Rangel RJM, Castro DS, Amorim MHC, Christoffel MM, Primo CC. Practice of insertion, maintenance and removal of peripheral inserted central catheter in neonates. J Res Fundam Care Online. 2019; 11(n.esp.):278-84. doi: http://dx.doi. org/10.9789/2175-5361.2019.v11i2.278-284

17. Organização Pan-Americana da Saúde. Centro Latino-Americano de Perinatologia, Saúde da Mulher e Reprodutiva. Prevenção de infecções relacionadas à assistência à saúde em neonatologia. Montevidéu: CLAP/SMR-OPS/OMS; 2016.

18. Curan GRF, Rossetto EG. Interventions to decrease catheter-associated bloodstream infections in newborns: an integrative review. Texto Contexto Enferm. 2017; 26(1):e5130015. doi: http:// dx.doi.org/10.1590/0104-07072017005130015

19. Morais APS, Façanha SMA, Rabelo SN, Siebra e Silva AV, Queiroz MVO, Chaves EMC. Non-pharmacological measures in the pain management in newborns: nursing care. Rev Rene. 2016; 17(3):43542. doi: http://dx.doi.org/10.15253/21756783.2016000300019

20. Kegler JJ, Paula CC, Neves ET, Jantsch LB. Pain management in the use of the peripherally inserted central catheter in newborns. Esc Anna Nery. 2016; 20(4):e20160099. doi: http://dx.doi. org/10.5935/1414-8145.20160099 